\title{
Aneurisma micótico en cayado aórtico por Aspergillus fumigatus: aportación de un caso y revisión en la literatura
}

\author{
M. R. BURÓN FERNÁNDEZ, M. J. ORUEZÁBAL MORENO'

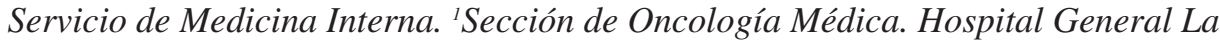 \\ Mancha Centro. Alcázar de San Juan, Ciudad Real
}

\section{RESUMEN}

Los aneurismas micóticos por Aspergillus son raros y suelen presentarse en el contexto de una aspergilosis pulmonar invasiva, bien por émbolos sépticos o por extensión directa desde los pulmones, por ello la localización más frecuente es en el cayado aórtico y aorta ascendente.

Se han descrito en la literatura 8 casos de aneurismas micóticos por Aspergillus spp. entre 1966 y 2000, siendo la localización más frecuente la aorta ascendente o el cayado aórtico. El Aspergillus fumigatus es la especie aislada con más frecuencia, afectando sobre todo a pacientes inmunodeprimidos.

El diagnóstico de un aneurisma micótico requiere una alta sospecha clínica, dada su rareza y la presencia de síntomas inespecíficos, siendo con frecuencia un hallazgo casual en el seno de un aspergilosis pulmonar invasiva.

Se presenta el caso de una paciente con un aneurisma micótico por $A$. fumigatus y revisamos los casos similares previamente divulgados .

PALABRAS CLAVE: Aneurisma micótico. Aspergillus.
MICOTIC ANEURISM IN AORTIC ARCH BY ASPERGILLUS FUMIGATUS: CONTRIBUTION OF A CASE AND REVISION IN LITERATURE

\section{ABSTRACT}

The micotic aneurisms by Aspergillus are rare and usually appear in the context of an invasive pulmonary aspergilosis, or by septicum embolism or direct extension from the lungs, for that reason the location the more frequents is in aortic arch and the ascending aorta.

8 cases of micotic aneurisms by Aspergillus spp. have been described in literature between 1966 and 2000, being the most frequent location the ascending aorta or the aortic arch. The Aspergillus fumigatus is the isolated species with more frequency, affecting mainly to patients undergoing inmunosupression. The diagnosis of a micotic aneurism requires a high clinical suspicion, given to its peculiarity and the presence of inespecific symptoms, being frequently an accidental finding in an invasive pulmonary aspergilosis.

The case of a patient with a micotic aneurism by A. fumigatus appears and we reviewed the similar cases previously disclosed.

KEY WORDS: Micotic aneurism. Aspergillus.

Burón Fernández MR, Oruezábal Moreno MJ. Aneurisma micótico en cayado aórtico por Aspergillus fumigatus: aportación de un caso y revisión en la literatura. An Med Interna (Madrid) 2005; 22: 437-440.

\section{INTRODUCCIÓN}

Los hongos del género Aspergillus, que excepcionalmente son responsables de patología en el paciente inmunocompetente, son agentes patógenos importantes en pacientes inmunocomprometidos, de hecho las diferentes formas clínicas que puede adoptar la enfermedad pulmonar dependen del mecanismo patogénico de acción, el estado inmunológico y la respuesta del huésped. En los últimos años la incidencia de la aspergilosis pulmonar invasiva ha ido en aumento en relación con un aumento de factores de riesgo como son la neutropenia $(<500 \mathrm{y}$, sobre todo, $<100$ células $\left./ 10^{6} / 1\right)$, neutropenia prolongada, tratamiento con esteroides, enfermedad del injerto contra huésped en los trasplantes de médula ósea, el rechazo agudo y la enfermedad por CMV en los trasplantados de órgano sólido y el sida avanzado. En la aspergilosis pulmonar invasora hay desarrollo miceliar en el parénquina pulmonar, con o sin angioinvasión, causando bronconeumonía, neumonía lobar, necrotizante e infarto hemorrágico. La infección puede diseminarse por vía hematógena o extenderse a estructuras contiguas, como los grandes vasos, produciendo hemorragias en ocasiones fatales.

Presentamos el caso de una rotura de un aneurisma micótico por Aspergillus spp. en un paciente con una aspergilosis pulmonar invasiva. 


\section{CASO APORTADO}

Paciente de 64 años con antecedentes personales de EPOC en tratamiento de larga evolución con broncodilatadores y corticoides orales.

Ingreso por tos, expectoración hemoptoica y dolor en hemitorax izquierdo. En la exploración física destacaba febricula y abolición del murmullo vesicular en dicho lado. El análisis de sangre mostró Hb 10,5 g/dL MCV 87 MHC 27 Leu 15.000 (N85\% L 20\%), resto normal. En la radiografía de tórax se observaba un infiltrado alveolar en lóbulo superior izquierdo. Ante estos hallazgos se inició tratamiento antibiotico empírico con ceftriaxona 1g/día. En los días siguientes persistía febricula, astenia, anorexia, tos y expectoración por lo que se le realizó una broncoscopia que no mostró alteraciones en el árbol bronquial. La citología del lavado bronquioalveolar fue negativa de malignidad y el cultivo para aerobios, anaerobios, hongos y micobacterias fue negativo. Unos días después se observó una leve mejoría y ante la ausencia de fiebre fue dado de alta con el mismo tratamiento antibiotico.

A los diez días, comenzó de nuevo con fiebre de $38^{\circ} \mathrm{C}$ y tuvo varios episodios de inestabilidad al adoptar la posición de bipedestación, obligandole a permanecer en decúbito. En la radiografía de torax persistía un infiltrado alveolar en el lóbulo superior izquierdo. Se tomaron hemocultivos que fueron negativos, y cultivos de esputo donde se aisló en dos ocasiones Aspergillus fumigatus, por lo que se inició tratamiento con anfotericina B a dosis de $1 \mathrm{mg} / \mathrm{kg} /$ día. En los días siguientes el paciente tuvo varios presíncopes al adoptar la bipedestación, sin alteración en la exploración neurológica ni cambios electrocardiográficos. Dos días después de iniciar el tratamiento antifúngico se realizó una TAC torácica (Fig. 1) donde se observó un infiltrado cavitado en el lóbulo superior izquierdo con amplia base de implantación pleural y en contacto con el cayado aórtico. Tras la administración de contraste se observó un area hiperdensa sacular de $5 \mathrm{~cm}$ de diametro máximo en contacto con el cayado aórtico. Ante la sospecha diagnóstica de un aneurisma micótico se realizó una arteriografía (Fig. 2) que confirmó la presencia de un aneurisma en cayado aórtico con rotura de su pared, que requirió implantar una endoprótesis Talent. En los días siguientes el paciente permaneció afebril con escasa tos y expectoración. A los 15 días del ingreso el paciente tuvo una hemoptisis masiva falleciendo inmediatamente.

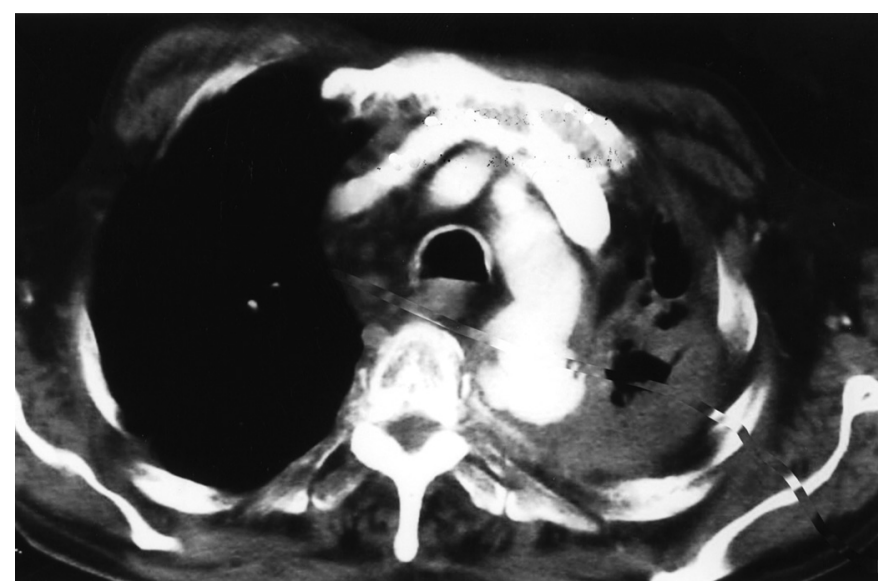

Fig. 1. TAC tórax. infiltrado cavitado en lóbulo superior izquierdo, con invasión de cayado aórtico. Aneurisma sacular de $5 \mathrm{~cm}$ en cayado aórtico.

\section{DISCUSIÓN}

Los aneurismas micóticos de la aorta son raros, y están producidos por émbolos sépticos que anidan en la pared arte-

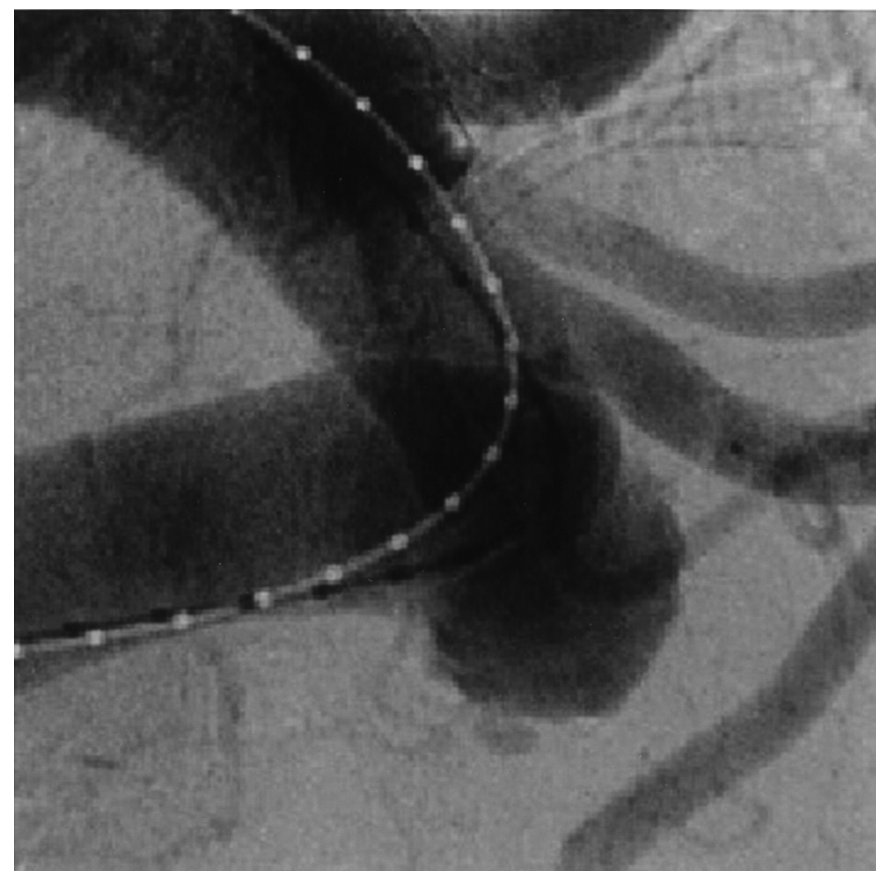

Fig. 2. Arteriografía. Aneurisma micótico de $5 \mathrm{~cm}$ en cayado aórtiCO.

rial originando una aortitis local y secundariamente la dilatación aneurismática (1).

El germen causal más frecuente es Staphylococcus aureus y epidermidis, aunque también se han aislado Salmonella, Clostridium septicum, Candida, etc. (2).

El Aspergillus spp. rara vez afecta la aorta o las válvulas cardiacas nativas aunque es frecuente que invada los vasos sanguíneos pulmonares durante el curso de la infección. Los casos descritos de afectación de las válvulas cardiacas se refieren a válvulas protésicas contaminadas por esporas que se propagan ampliamente siendo frecuente la implantación directa (3).

El desarrollo de la infección depende del estado inmunológico del paciente, ya que los macrófagos ingieren y degradan las esporas impidiendo la diseminación de las hifas. Por ello, la invasión tisular se observa casi exclusivamente en pacientes inmunodeprimidos, tales como los que sufren neutropenia prolongada primaria o secundaria a tratamientos citotóxicos, trasplante renal, hepático o cardiaco y médula ósea, dosis elevadas de corticoides, o enfermedad granulomatosa crónica (4-8).

El Aspergillus fumigatus supone el $90 \%$ de las especies aisladas en los casos de aspergilosis invasiva, seguido por $A$. flavus. Otras especies como Aspergillus terreus son infrecuentes patógenos humanos, aunque está aumentando su aislamiento en pacientes inmunodeprimidos tras trasplante cardiaco (9).

Se han descrito en la literatura 8 casos de aneurismas micóticos por Aspergillus spp. entre 1966 y 2000 (10-17), afectando en un caso el seno de Valsalva, en cuatro casos la aorta ascendente o el cayado aórtico, y en tres la aorta descendente (Tabla I). El Aspergillus fumigatus fue la especie aislada con más frecuencia, seguida del Aspergillus flavus, terreus y niger. El factor predisponente que predominó fue la leucemia, en dos casos, y trasplante cardiaco y renal en otros dos, destacando una infección en un paciente inmunocompetente, de hecho se han comunicado casos de aspergilosis invasiva en 
TABLA I

CARACTERÍSTICAS DE LOS CASOS DE ANEURISMAS MICÓTICOS POR ASPERGILLUS SPP. PUBLICADOS EN LA LITERATURA 1966-2000

\begin{tabular}{lcccc}
\hline Referencia & Edad/sexo & Antecedentes & Especie aspergillus & Localizacion aneurisma \\
\hline $1(10)$ & $39 / \mathrm{V}$ & Alcoholismo cirrosis & A. fumigatus & Seno valsalva \\
$2(11)$ & $51 / \mathrm{V}$ & TBC & A. fumigatus & Cayado aórtico \\
$3(12)$ & $54 / \mathrm{M}$ & Trasplante renal & A. fumigatus & Aorta ascendente \\
$4(13)$ & $54 / \mathrm{V}$ & Diabetes mellitus & A. fumigatus & Aorta descendente \\
$5(14)$ & $51 / \mathrm{V}$ & Trasplante cardiaco & A. fumigatus & Aorta ascendente \\
$6(15)$ & $11 / \mathrm{M}$ & LMA & A. flavus & Aorta descendente \\
$7(16)$ & $74 / \mathrm{M}$ & EPOC & A. niger & Aorta infrarrenal \\
$8(17)$ & $11 / \mathrm{V}$ & LMA & A. terreus & Cayado aórtico
\end{tabular}

sujetos aparentemente sanos; entre ellos, algún caso asociado a cirrosis y a virus de influenza A $(18,19)$.

La sospecha clínica es esencial para el diagnóstico ya que los datos clínicos son inespecíficos, siendo los más frecuentes fiebre, dolor torácico, presíncopes, y hemoptisis (20). La tos puede estar presente o no y raramente es productiva.

En la radiografía de tórax rara vez se observan signos que hagan sospechar la existencia de una dilatación aneurismática y la mayoría de las veces se diagnostica en la TAC solicitada por una evolución tórpida o para detectar signos precoces de infección por Aspergillus. La arteriografía permitirá confirmar el diagnóstico a la vez que demostrar la existencia de fugas de contraste por rotura de la pared, permitiendo la implantación de endoprótesis y una rápida resolución de la lesión aórtica con escaso riesgo postoperatorio (21).

Para realizar el diagnóstico definitivo de aspergilosis invasiva debe demostrarse invasión parenquimatosa, aunque el aislamiento de Aspergillus de las secreciones respiratorias en pacientes con inmunosupresión es altamente predictivo de aspergilosis invasiva, y debe considerarse el tratamiento antifúngico sin necesidad de diagnóstico tisular $(22,23)$.
La administración intravenosa de anfotericina B es el tratamiento de elección siendo la dosis más apropiada de 1 $\mathrm{mg} / \mathrm{kg} /$ día. El itraconazol en estudios in vitro mantiene la mismas tasas de actividad que la anfotericina B aunque debido a su absorción erratica se debe monitorizar las dosis plasmáticas para garantizar la eficacia terapeutica, dificultando su utilización. El voriconazol es actualmente otra opción terapéutica en pacientes con mala tolerancia o respuesta a la anfotericina B (24).

$\mathrm{El}$ aneurisma micótico rara vez requiere tratamiento quirúrgico dado el pronóstico desfavorable y la alta morbimortalidad perioperatoria, siendo preferible la implantación de una endoprótesis transfemoral que permitirá en poco tiempo y con escaso riesgo de isquemia medular resolver una lesión por sí misma desastrosa.

En resumen, el diagnóstico de un aneurisma micótico requiere una alta sospecha clínica, dada su rareza y la presencia de síntomas inespecíficos, siendo con frecuencia un hallazgo casual en el seno de un aspergilosis pulmonar invasiva. Aunque la mortalidad es elevada la utilización de anfotericina $\mathrm{B}$ combinado con endoprotesis, puede evitar la rotura aortica y la progresión de la invasión vascular.

\section{Bibliografía}

1. Stemmet F, Davies JQ, Von Oppell UO. Postpneumonectomy aortic arch mycotic aneurysm. Ann Thorac Surg 2001; 71: 1030-2.

2. Enia F, Di Stefano G, Floresta AM, Matassa C. New etiologies responsible for infective endocarditis with negative blood cultures. Ital Heart J Suppl 2005; 6: 128-34.

3. El-Hamamsy I, Durrleman N, Stevens LM, Cartier R, Pellerin M, Perrault LP, Carrier M. A cluster of cases of Aspergillus endocarditis after cardiac surgery. Ann Thorac Surg 2004; 77: 2184-6.

4. Willemsen P, De Roover D, Kockx M, Gerard Y. Mycotic common carotid artery aneurysm in an immunosuppressed pediatric patient: case report. J Vasc Surg 1997; 25: 784-5.

5. Garrido J, Labrador PJ, Lerma L, Heras M, García P, Bondia A, Corba- cho L, Tabernero M. Vascular Aspergillus infection in two recipients of kidneys from the same donor. Nefrología 2004; 24 (Supl. 3): 30-4.

6. Levin T, Suh B, Beltramo D, Samuel R. Aspergillus mediastinitis following orthotopic heart transplantation: case report and review of the literature. Transpl Infect Dis 2004; 6: 129-31.

7. Ergin F, Arslan H, Azap A, Demirhan B, Karakayali H, Haberal M. Invasive aspergillosis in solid-organ transplantation: report of eight cases and review of the literature. Transpl Int 2003; 16: 280-6.

8. Hachem RY, Kontoyiannis DP, Boktour MR, Afif C, Cooksley C, Bodey GP, et al. Aspergillus terreus: an emerging amphotericin B-resistant opportunistic mold in patients with hematologic malignancies. Cancer 2004; 101: 1594-600. 
9. Montoya JG, Chaparro SV, Celis D, Cortes JA, Leung AN, Robbins $\mathrm{RC}$, Stevens DA. Invasive aspergillosis in the setting of cardiac transplantation. Clin Infect Dis 2003; 37 (Supl. 3): S281-92

10. Iwen PC, Rupp ME, Langnas AN, Reed EC, Hinrichs SH. Invasive pulmonary aspergillosis due to Aspergillus terreus: 12-year experience and review of the literature. Clin Infect Dis 1998; 26: 1092-1097.

11. Caplan HI, Frisch E, Houghton JD, Climo MS, Natsios GA. Aspergillus fumigatus endocarditis: report of a case diagnosed during life. Ann Intern Med 1968; 68: 378-385.

12. Burke BJ, Storring FK, Parry TE. Disseminated aspergillosis. Thorax 1970; 25: 702-707

13. Myerowitz RL, Friedman R, Grossman WL. "Mycotic aneurysm" of the aorta due to Aspergillus fumigatus. Am J Clin Pathol 1971; 55: 241246.

14. Rose HD, Stuart JL. Mycotic aneurysm of the thoracic aorta caused by Aspergillus fumigatus. Chest 1976; 70: 81-84.

15. Byl B, Jacobs F, Antoine M, et al. Mediastinitis caused by Aspergillus fumigatus with ruptured aortic pseudoaneurysm in a heart transplant recipient: case study. Heart Lung 1993; 22: 145-147.

16. Wells WJ, Fox AH, Theodore PR, Ross LA, Stanley P, Starnes VA. Aspergillosis of the posterior mediastinum. Ann Thorac Surg 1994; 57 : 1240-1243.

17. Smith FC, Rees E, Elliott TS, Shearman CP. A hazard of immunosuppression: Aspergillus niger infection of abdominal aortic aneurysm. Eur
J Vasc Surg 1994; 8: 369-371

18. Rutter P, Wolfe J. Infected or mycotic aneurysms. In: Horrocks M, ed. Arterial aneurysms: diagnosis and management. Oxford: ButterworthHeinemann, 1995: 221-230.

19. Brown E, Freedman S, Arbeit R, Come S. Invasive pulmonary aspergillosis in an apparently nonimmunocompromised host. Am J Med 1980; 69: 624-627.

20. Sanchis Minguez C, Crespo Liern ML, Perez Enguix D, Compte Torrero L, Montero Benzo R. Cardiac tamponade caused by aortic pseudoaneurysm with fistula in the right ventricle Rev Esp Anestesiol Reanim 2003; 50: 521-5.

21. Sole A, Morant P, Salavert M, Peman J, Morales P; Valencia Lung Transplant Group. Aspergillus infections in lung transplant recipients: risk factors and outcome. Clin Microbiol Infect 2005; 11: 359-65.

22. MRI of mycotic sinus of valsalva pseudoaneurysm secondary to Aspergillus pericarditis. AJR Am J Roentgenol 2005; 184 (Supl. 3): S25-7

23. Fink-Josephi G, Gutiérrez-Vogel S, Dena-Espinoza E, Hurtado-López LM, Zaldivar-Ramírez FR. Tratamiento endovascular de los aneurismas de la aorta abdominal en pacientes mayores de 60 años: morbimortalidad perioperatoria. Cir Gen 2002; 24: 32-37.

24. Wieland T, Liebold A, Jagiello M, Retzl G, Birnbaum DE. Superiority of voriconazole over amphotericin B in the treatment of invasive aspergillosis after heart transplantation. J Heart Lung Transplant 2005; 24 : 102-4. 